Musées, Patrimoine et Culture scientifiques et techniques

$108 \mid 2006$

novembre-décembre 2006

\title{
Entre art et science :
}

Les leçons d'une création pour planétarium numérique

Jean-François Desmarchelier et Jacques Roux

\section{(2) OpenEdition}

Édition électronique

URL : http://journals.openedition.org/ocim/1320

DOI : $10.4000 /$ ocim. 1320

ISSN : 2108-646X

Éditeur

OCIM

\section{Édition imprimée}

Date de publication : 25 novembre 2006

ISSN : 0994-1908

\section{Référence électronique}

Jean-François Desmarchelier et Jacques Roux, "Entre art et science : », La Lettre de l'OCIM [En ligne], 108 | 2006, mis en ligne le 12 mars 2014, consulté le 21 avril 2019. URL : http://

journals.openedition.org/ocim/1320 ; DOI : 10.4000/ocim.1320 


\section{Entre art et science :}

\section{Les leçons d'une création pour planétarium numérique}

\section{Jean-François Desmarchelier et Jacques Roux *}

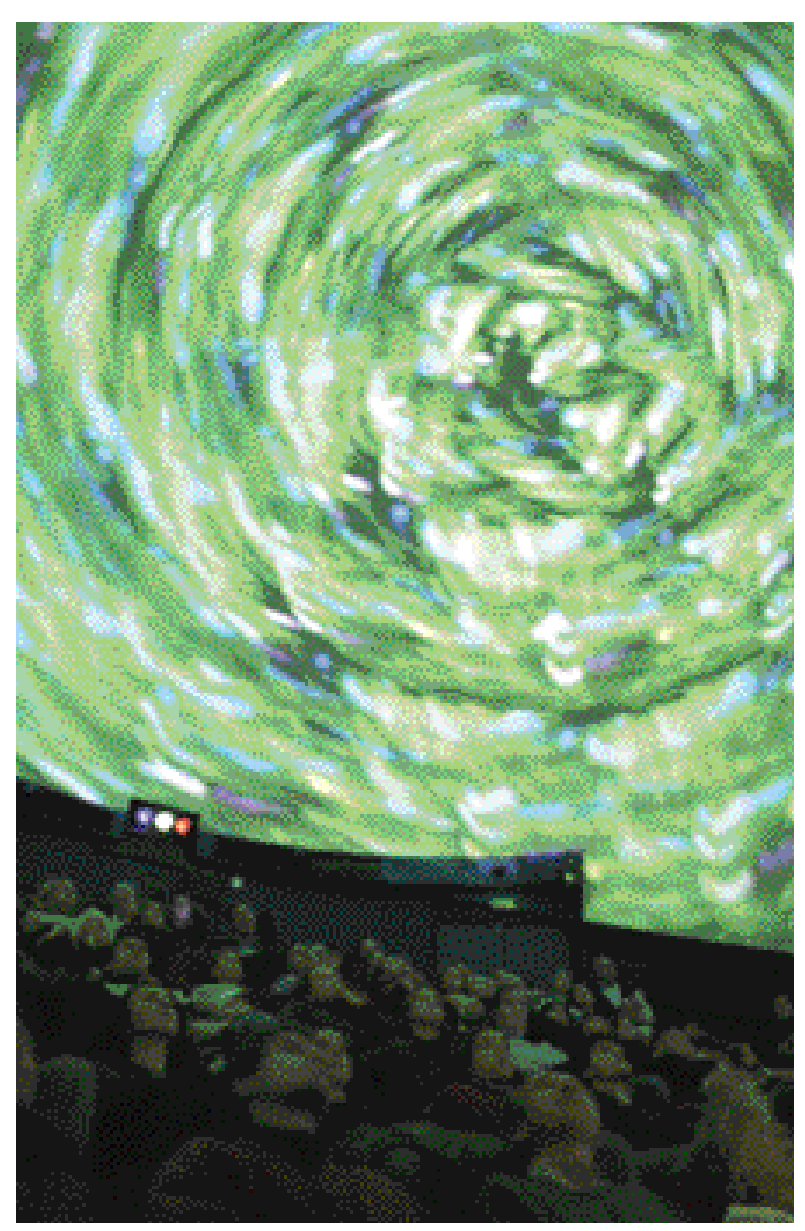

Vibrato, la résonance de l'Univers: des spectateurs entourés, immergés dans l'image. (c) Valéry Maillot

* Jean-François Desmarchelier est réalisateur, scénographe et chargé de mission en culture scientifique

jfdesmar@hotmail.com

Jacques Roux est auteur, metteur en scène et réalisateur jacques.roux@univ-st-etienne.fr
À partir de la création d'un spectacle de planétarium basé sur une fiction qui plonge le spectateur au cœur de l'activité du chercheur en Astronomie - lui faisant partager ses interrogations et ses doutes les auteurs mènent une réflexion sur le renouveau possible de ce type de spectacle en montrant comment cette expérience émotionnelle d'exploration scientifique peut accompagner le public dans sa découverte de la science en train de se faire.

Depuis une dizaine d'années, le milieu des planétariums est saisi par la révolution du numérique. Un système de multi-diffusion pleine voûte immerge le spectateur dans la dynamique de l'image et lui donne des sensations qui concurrencent maintenant directement celles qui ont jusque-là été permises par des salles équipées avec des écrans géants ou circulaires (comme les techniques IMAX). C'est à cette expérience sensible d'une séance de projection dans un planétarium équipé d'une telle technique numérique (l'Astronef à Saint-Étienne) que nous pouvons dater notre projet de créer un spectacle à base cosmologique dédicacé à ce nouveau type de théâtre immersif : Vibrato, la résonance de l'Univers.

Cette création, entre théâtre et cinéma, s'est appuyée sur les compétences de trois groupes : notre structure, ART'M créateurs associés, à l'origine compagnie de théâtre (en particulier théâtre de science depuis quelques années), l'équipe de l'Astronef (dirigé à l'époque par Ghaouti Hansali), et 
Le résumé de l'histoire

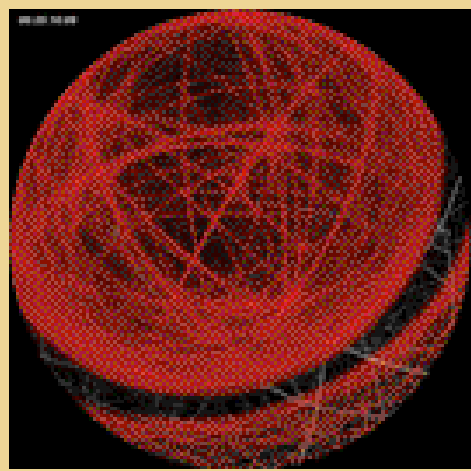

Le Big Bang présenté non comme un point de départ mais comme un phénomène qui ne cesse de commencer. ○ APSIDE

Tout commence dans un observatoire virtuel, quelque part dans le désert d'Atacama au Chili. Paul, Frank et leur assistante d'un soir Léa, effectuent une mission de routine dans leur observatoire virtuel. Cet observatoire est assez particulier car il leur permet non seulement de visualiser le ciel tel que le verrait n'importe quel observateur qui lèverait la tête dans cette partie du monde, mais aussi d'accéder simultanément à toutes les images et informations fournies par les télescopes installés sur Terre ou dans l'Espace. Cet observatoire virtuel, s'il existait, ferait rêver plus d'un astronome professionnel pour qui l'observation des étoiles est essentiellement devenue l'analyse de données sur des ordinateurs, dans des bureaux...

Ce soir là, les trois chercheurs sont principalement attachés à la recherche des afterglows, sortes de rayonnements lumineux provenant du fin fond de l'Univers. Les premières heures de la nuit défilent, semblables à tant d'autres, jusqu'à ce qu'un phénomène lumineux de très forte intensité attire leur attention. Paul décide alors de passer en mode de vision immersive, le dispositif leur permettant de se déplacer dans les images de l'Univers « comme sills y étaient ». C'est alors que limpensable se produit. Le virtuel prend le pas sur la réalité et les scientifiques se retrouvent happés, absorbés par un gigantesque gouffre qui a tout d'un trou noir... Au-delà de l'impossible, un bain mystérieux de brins de lumières et de mondes multicolores évoquera à Léa la théorie des cordes et des multivers. Alors qu'ils auraient pu perdre tout espoir de " retour », les trois héros entrent maintenant en résonance avec les premiers instants de l'Univers! Au gré du refroidissement du rayonnement fossile, ils vont vivre l'apparition des premières étoiles, la structuration de l'Univers, la formation des galaxies...

Après un dernier flash lumineux, ils se retrouvent à leur point de départ, dans leur observatoire suréquipé... qui n’a rien enregistré ! Était-ce un rêve ? Une hallucination collective ? Quoi quill en soit, ce n'est que longtemps après que Paul fut capable de nous raconter leur histoire... les chercheurs de l'Observatoire de Lyon (CRALCNRS), notamment Bruno Guiderdoni et Isabelle Vauglin qui, à toutes les étapes du projet, ont assuré leur rôle de conseillers scientifiques de Vibrato. La version «vivante », avec présence des comédiens sous la voûte, a été programmée dans le cadre du festival Scènes de Méninges et les cinq représentations ont fait salle comble. Depuis février 2006, la version « film » est programmée à l'Astronef. C'est cette création et sa philosophie que nous nous attacherons à décrire dans un premier temps.

Le film, désormais disponible pour toute salle équipée du système de projection pleine voûte, a été visionné, expertisé par des responsables de planétariums ou par des experts missionnés. Les retours que nous avons eus nous ont permis de mieux cerner les enjeux, les obligations, les besoins et les envies des équipes qui ont en charge le fonctionnement de ces lieux de culture scientifique en pleine évolution. La mission historique des planétariums, du moins en France, est d'éduquer le spectateur en lui montrant le ciel. C'est dans ce cadre que les ressources sont mobilisées et perfectionnées. Des outils très précis montrent sur la voûte les étoiles ou d'autres phénomènes visibles à partir de la Terre comme les planètes du système solaire - et des équipes d'animateurs (souvent des passionnés d'Astronomie) construisent de véritables «leçons de choses » astronomiques à un public sensé ignorer tout ou partie de son propre ciel. Le défi des nouvelles technologies dans le milieu des planétariums vient $\mathrm{du}$ fait qu'il est maintenant possible de faire autre chose dans ces salles. Dès lors, des questions nouvelles se posent à chaque nouvelle création : accepte-t-on une banalisation des salles, comme certaines à l'étranger (Athènes, Londres), qui abandonnent complètement toute référence à l'Astronomie pour devenir de simples salles de spectacle ? Ou bien relève-t-on de nouveaux défis dans la création de spectacles astronomiques? Jusqu'où peut-on aller dans la relation à la science et aux scientifiques, dès lors qu'il est possible de créer des aventures qui vont bien au-delà de la seule présentation du ciel ? C'est dans un tel contexte que l'expérience de Vibrato se poursuit, et c'est cet aspect que nous allons maintenant développer.

\section{Un spectacle à part entière}

Vibrato se raconte comme une histoire. La narration, la mise en histoire, nous l'avons abordée avec les ressources propres à cet outil formidable que constitue, 
pour une équipe de théâtre, un planétarium doté de l'équipement de projection pleine voûte. Ce média, que nous avons découvert avec Vibrato mais que nous allons approfondir dans notre prochaine création, se trouve posséder des caractères tenant à la fois de la salle de cinéma grand écran et de la salle de théâtre. Nous avons d'ailleurs créé Vibrato en tant que spectacle vivant, avec présence des comédiens devant le public. Dans le cas de la version sans acteurs (juste leurs voix), il ne s'agit pas à proprement parler d'un film mais vraiment d'un spectacle dans sa dimension sensible. Le spectateur n'est pas devant une image sonorisée, devant une représentation, il est entouré, immergé dans l'image. Le système de diffusion sonore en 5.1 permet une précision et une spatialisation des voix, de la musique, des bruitages. Nous avons mobilisé ces dernières ressources pour donner au spectateur la sensation de se trouver au milieu des personnages, dans leur monde de scientifiques en train de travailler. Du coup, les images des phénomènes astronomiques et cosmologiques qui nourrissent l'exploration de Vibrato sont d'une telle force suggestive que ce n'est pas intellectuellement mais de manière sensible que le spectateur plonge dans un trou noir, emprunte un " trou de ver », assiste au Big Bang ou à la collision de deux galaxies.

\section{Le mixage des sources d'images}

À l'inverse d'autres créations pour vidéo pleine voûte que nous avons pu visionner (nous pensons notamment à Kaluoka'Hina de Peter Popp, du groupe Softmachine de Munich), Vibrato collectionne et rassemble des images d'origines très différentes. Il ne s'agit pas d'une production homogène, mais plutôt d'une intégration de différentes sources d'images : infographie, vidéo, simulations scientifiques, photographies astronomiques ou artistiques. Bien évidemment, cet assemblage d'images de sources différentes a demandé un important travail de synthèse, de montage, d'assemblage pour conserver la cohérence artistique de l'ensemble. Cette cohérence est aussi le résultat d'un travail d'équipe. Plusieurs compétences doivent ici s'associer pour aboutir à un résultat qui se tienne. Ceci est particulièrement sensible dans les moments où des choix doivent se faire, par rapport aux possibilités techniques des outils ou aux contraintes de temps.

Nous pensons que ce qui peut apparaître comme un défaut d'homogénéité de traitement de l'image est au contraire, dans le cas de Vibrato, une qualité attachée aux possibilités du média qui était à notre disposition. Qui plus est, une qualité qui prend en charge et met en valeur le propos que nous défendons dans le spectacle. En effet, restituer l'effort humain pour comprendre le cosmos, s'accommode mieux d'une diversité des sources d'information, des données, des images. En fonction des étapes du «voyage » dans Vibrato, le spectateur connaît une succession d'épreuves esthétiques et émotives et non pas une narration lissée dans un univers homogène et parfait. L'ouverture à la connaissance n'est pas ici piégée par un émerveillement qui serait saturé par l'image. Ce sont des ouvertures successives aux questions et à la curiosité, qui s'inscrivent au cœur d'une expérience émotionnelle. Vibrato rejoint ici, aux antipodes d'un pédagogisme étroit, les plus récentes recherches pédagogiques sur l'importance de l'affectif dans les processus de transmission et d'acquisition des savoirs.

\section{La science montrée comme une aventure humaine}

Durant le voyage de Vibrato, nous sommes transportés dans le cosmos, au même titre que les héros. Nous sommes avec ces trois chercheurs, nous sommes témoins de leur travail, nous les observons, nous les écoutons, nous sommes au milieu d'eux, nous vivons leurs émotions, leurs déceptions, leurs espoirs, leurs surprises, leurs angoisses. Ils ne sont pas loin du spectateur, ce sont des humains. Le passage à la fiction, sa crédibilité, s'adosse ici directement sur ce que nous pourrions appeler : "l'expérience de l'image ». Le principe suivi dans Vibrato, c'est qu'observer, d'une certaine manière, c'est voyager dans le phénomène qu'on observe. Et cette idée est d'autant plus forte, et d'autant plus partageable aujourd'hui, que les chercheurs disposent, dans le cadre de leurs travaux, d'images de simulation, qui font voir " comme si on y était ». Il est alors possible de faire sentir au spectateur ce qui est de l'ordre affectif, émotionnel, dans l'activité du chercheur qui observe et qui cherche à comprendre. Cette expérience n'est d'ailleurs pas si différente de celle de l'humain qui fait un grand voyage, loin de ses bases, loin de ce qu'il connaît. Cette expérience émotionnelle de la découverte et de l'exploration prend le pas, dans Vibrato, sur le discours intellectuel de la raison scientifique. Ce qui implique le spectateur, de tout âge et de toute culture, dans l'histoire de Vibrato et dans son dénouement, c'est le maintien d'une intrigue, d'un suspens. Cette envie d'aller plus loin, de découvrir toujours et encore, de prendre des risques pour y parvenir, conduit jusqu’à la chute finale, qui résonne comme un appel à la sagesse, à la modestie, à une certaine philosophie du respect de l'Univers. 


\section{La diversité des sources d'images}

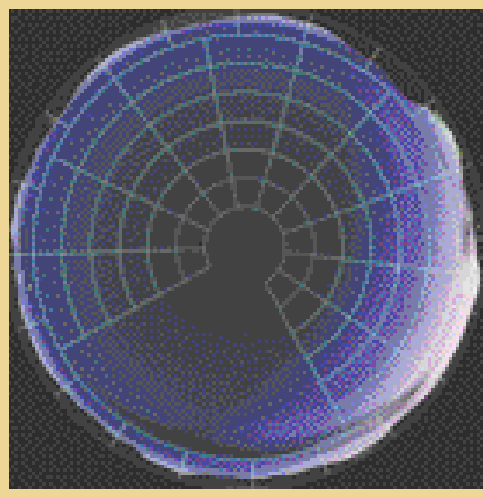

Une image du temps réel : l'observatoire virtuel du début et de la fin du film (c) Astronef

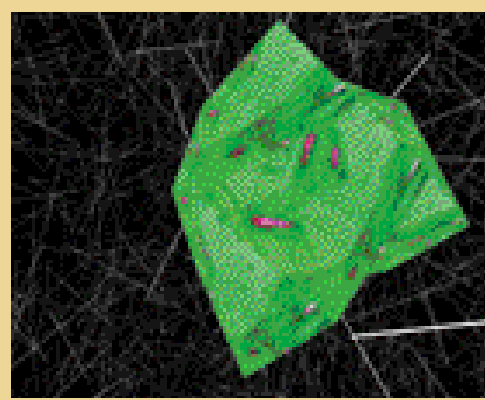

Une image d'infographie : un multivers dans un bain de cordes (c) APSIDE/ART'M

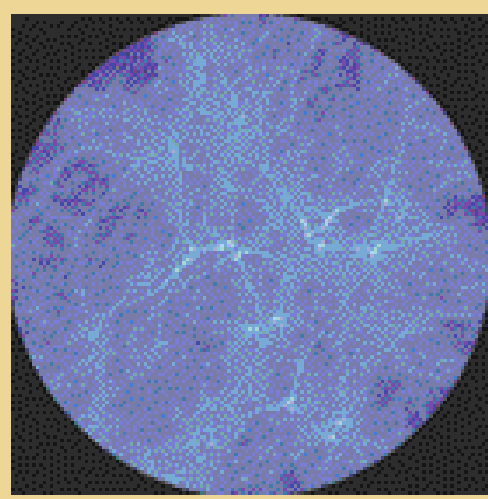

Une image de science : une simulation des premiers temps de l'Univers (c) Dapnia-CEA Saclay/Yann Rasera
Vibrato est constitué de plusieurs types d'images qui n'ont pas toutes le même statut, certaines sont totalement créées alors que d'autres proviennent de la science.

\section{Le temps réel}

Les planétariums numériques sont équipés de bases de données dans lesquelles il est possible de se déplacer en temps réel. Ce dispositif permet de se positionner en un lieu et une date donnés dans notre univers proche. Le dispositif permet également de générer des caractères simples, d’insérer des images ou des vidéos. Le temps réel nous a permis de réaliser plusieurs séquences du film (déplacements dans les étoiles, création de l'observatoire virtuel...). Il est à noter que le spectacle a été, dans un premier temps, entièrement attaché au fonctionnement de ce dispositif : « lancer » le film revenait à « lancer » un script de plus de 2500 lignes de commandes, qui en temps réel générait les étoiles, les inscriptions, les graphiques et « appelait » les vidéos, les images fixes et tous les éléments constituant le film.

\section{Les vidéos}

À l'inverse de certaines créations traditionnelles pour planétariums, Vibrato contient un grand nombre de séquences d'infographie et d'animations 3D à thème cosmologique (traversée de nébuleuse, trou noir, Big Bang...). La définition du format DV ne permet pas de projeter des images sur la voûte sans une importante pixellisation. Nous avons néanmoins utilisé cette technique pour créer nos multivers. Les images sont abstraites et texturent des formes et des volumes créés en infographie. La souplesse de la vidéo et du montage virtuel, moins gourmands en temps de calculs que les rendus en 3D, rend possible des phases d'expérimentations.

\section{Les images de science}

Les photographies : le nombre de photographies astronomiques utilisées dans Vibrato n'est pas très important, mais à nouveau, le dispositif nous a permis de créer certaines séquences animées au départ d'une simple image fixe. Ainsi, avec un léger traitement, un simple zoom vers le Grand Nuage de Magellan donne une véritable illusion de travelling avant...

Les simulations : le dernier grand groupe d'images animées utilisées ont directement été créées par des équipes de recherche (groupe Horizon, CNRS, CEA, NASA...). Certaines nous ont été confiées par les scientifiques qui ont collaboré à l'écriture du scénario. La plupart des simulations sont d'une telle qualité, qu'il faudrait un travail considérable à des infographistes pour recréer des images ressemblant à ces modélisations. En effet, chaque mouvement, chaque trajectoire, chaque nuage de gaz ne résulte pas d'un travail graphique, mais de la résolution d'équations. Certaines simulations ont nécessité plusieurs mois de calcul sur des stations de travail extrêmement performantes.

Notons enfin le véritable échange qui s’est opéré avec les scientifiques. Lorsqu’ils ont vu leurs images de science pour la première fois sur la voûte du planétarium, ils se sont rendus compte que ce lieu de « monstration » d'images de science pouvait devenir un nouveau site d'observation : enfin ils pouvaient observer leurs simulations en 3D sur un écran géant et non plus sur un moniteur d'ordinateur, en 2D... 


\section{Un spectacle ouvert aux sens}

L'aventure scientifique dans Vibrato n'est pas engoncée dans un pédagogisme étroit. La science du cosmos n'est pas réduite à des résultats d'observation ou à des controverses théoriques entre savants : c'est la recherche en action, en train de se faire, qui en constitue le cœur. Ce qui nous fait dire que Vibrato, spectacle généreux en émotion suscitée par l'histoire racontée et la beauté des images présentées, est aussi un spectacle qui accompagne le public dans sa découverte de la science. Un public très ouvert, parce que chaque spectateur, chaque tranche d'âge, chaque génération, porte avec lui ou elle une relation de connaissance spécifique à ce qu'est l'Univers et ce que peut en dire la science aujourd'hui. Nous avons vu des jeunes enfants de moins de dix ans se lever de leur fauteuil quand les chercheurs aperçoivent, au terme de leur périple, le Soleil et la Terre. Nous avons aussi en tête la qualité et la pertinence des questions du public après les séances de projection.

Le langage, parfois très technique, attribué aux personnages a une double fonction. D'une part, il ancre l'histoire dans un milieu, différent du monde habituel des spectateurs (un monde de spécialistes, d'experts, a un langage spécifique, et il est normal qu'on

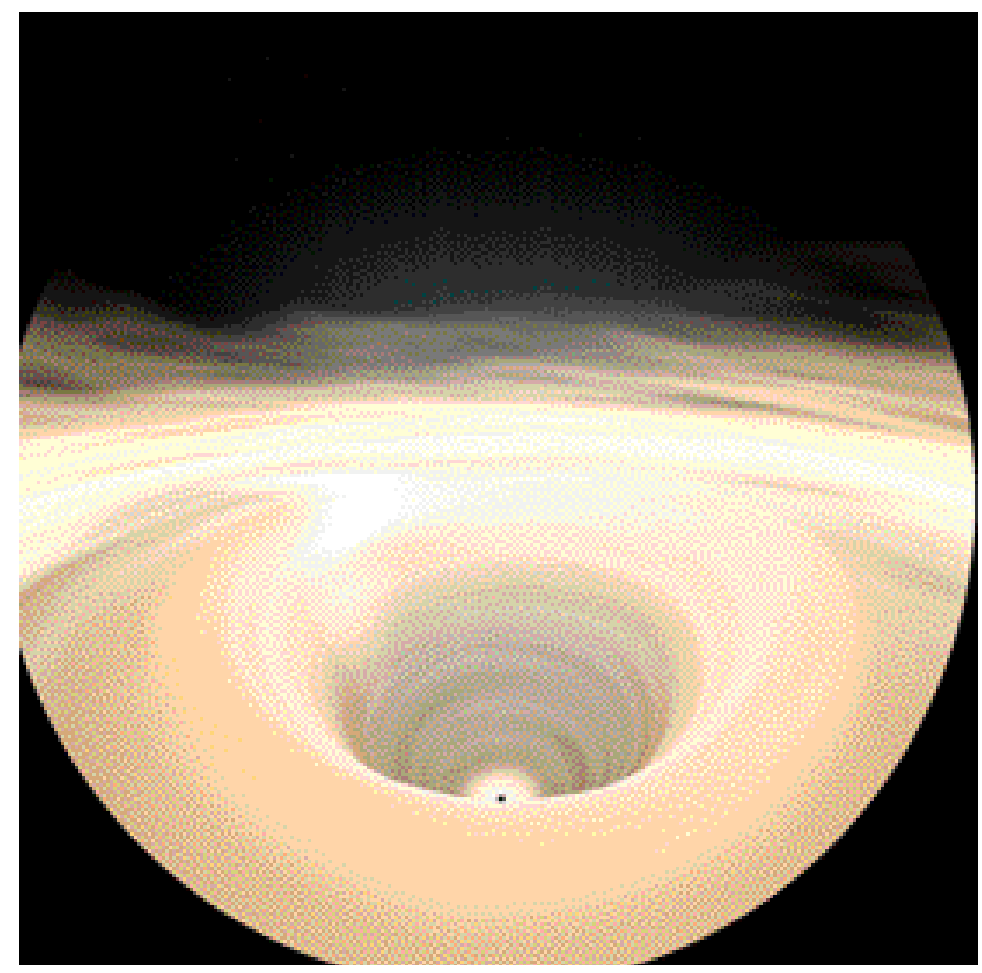

Des spectateurs plongés dans un trou noir et... () APSIDE/Marc Dalmas ne le comprenne pas entièrement quand ils parlent entre eux) ; d'autre part, il transmet au spectateur averti des données sur lesquelles il peut s'appuyer. Mais, chaque phrase n'a pas un but pédagogique, chaque réplique n'est pas l'occasion de " placer » un contenu scientifique. Dans Vibrato, l'essentiel est de « rentrer » dans l'histoire, dans son histoire. Loin de nous la volonté de distiller à tout prix un certain nombre de notions, en suivant une sorte de cahier des charges imposé par un programme... En revanche, Vibrato peut donner envie au spectateur de se renseigner pour en savoir plus, et nous avons élaboré avec l'équipe de l'Astronef un matériel spécifique d'accompagnement du spectateur, sous la forme d'un livret apportant des premières réponses sur certains mots-clés du spectacle, et une liste de sites Internet permettant d'accéder à une bonne documentation.

\section{L'avis des spécialistes}

Les premières présentations de Vibrato devant des professionnels des planétariums, susceptibles d'assurer à ce spectacle une diffusion nationale, voire internationale, sont particulièrement contrastées. Alors que tous sont unanimes pour souligner le caractère original de cette création dans l'ensemble

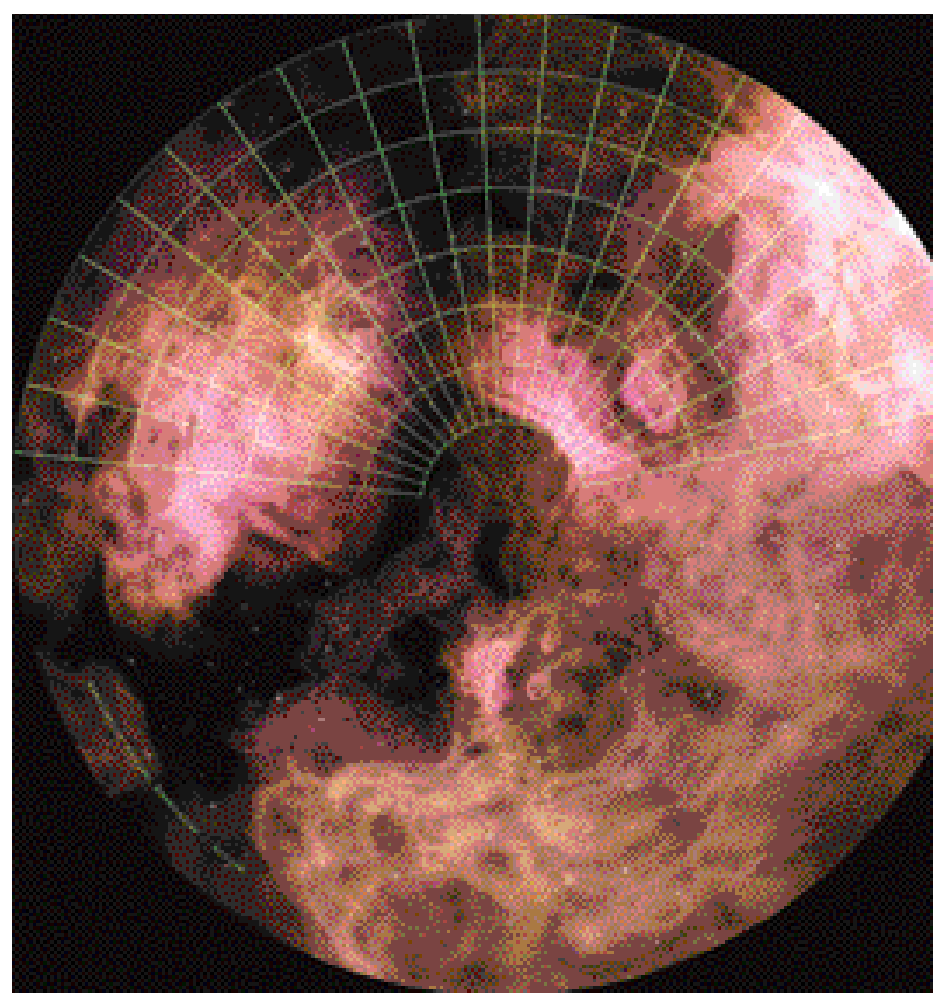

... empruntant la traversée du Grand Nuage de Magellan (c) APSIDE 
des propositions destinées aux planétariums, certains experts, appelés à donner un avis de scientifiques pour en valider le contenu, se sont déclarés gênés, voire indisposés par ce qu'ils désignent comme une confusion entre documentation réaliste du travail des scientifiques et fiction, voire science-fiction. Reprenant cette thématique, un astrophysicien réputé nous a demandé d'inscrire un marqueur au moment où le spectacle bascule du documentaire à la fiction. Pour d'autres, la mention de certains noms réels peut prêter à confusion, de même que la figuration d'une

\section{Réalité, réollisme, réalisotion}

Plusieurs experts scientifiques nous ont mis en garde face à la confusion que Vibrato induirait chez le spectateur non spécialiste (en cosmologie), en ne marquant pas la rupture entre une première (réalité) et une deuxième partie (fiction). Nous pensons qu'il s'agit plus d'une rupture dans la réalisation que dans le statut de fiction du film. Pendant les douze premières minutes, l'histoire se passe dans un observatoire, que nous avons localisé dans le désert d'Atacama. Des chercheurs sont au travail, devant des ordinateurs et des écrans de contrôle. L'ensemble peut paraître plausible, d'autant que le film débute avec un narrateur en voix-off, qui pourrait renvoyer à un commentaire de documentaire. Cette première étape met en jeu des effets de réel. Dans un deuxième temps, les héros (et les spectateurs) sont embarqués dans les images. Le doute est semé entre voyage virtuel dans les images, et voyage réel dans le cosmos. Les images, les dialogues font disparaître le réalisme de l'histoire.

C'est cette différence de traitement de l'histoire et des images qui a suggéré à certains scientifiques qu'il s'agissait du passage du réel à la fiction. Nous pensons que cette interprétation, qui vise à différencier la fiction de la réalité dans Vibrato en se basant sur des éléments de réalisation, résulte d'une confusion entre réalité et réalisme. Le réalisme cinématographique n'est pas la réalité, il en donne seulement l'impression... La réalité c'est que Vibrato est une fiction du début à la fin. L'histoire, les personnages sont totalement inventés. Mais la première partie du film est traitée sur un mode plus réaliste. Il n'y aurait donc pas de sens à marquer une quelconque rupture entre première et deuxième partie, entre réalité et fiction. hypothèse (la théorie des cordes) peut aussi induire le spectateur en erreur, dans la mesure où il n'a pas les moyens de se faire un jugement et de replacer cette théorie dans l'ensemble des théories actuellement en controverse. Nous avons tenu compte de certaines de ces remarques (par exemple de modifier l'avertissement initial qui signifie clairement au spectateur qu'il s'agit d'une fiction), mais nous avons également souhaité respecter une œuvre qui s'impose dorénavant dans son intégrité ${ }^{(1)}$.

Vibrato est une fiction, c'est un voyage imaginaire dans le cosmos. Mais c'est une fiction qui a la particularité d'être inspirée, d'être nourrie, d'être adossée à des éléments qui correspondent à un certain « état de l'art » de la science d'aujourd'hui dans les domaines astrophysiques et cosmologiques. Il y a dans Vibrato une collection d'éléments, de contenus, qui se rapportent à différents pans de l'activité de recherche scientifique contemporaine dans ces domaines. Le spectacle commence avec des chercheurs impliqués dans un réseau de surveillance des rayonnements de très hautes énergies venant du fond de l'Univers (les sursauts gamma et les afterglows). Mais très vite leurs observations les conduisent à découvrir des phénomènes beaucoup plus lointains et hypothétiques (les trous noirs, le Big Bang, les multivers, la théorie des cordes, l'univers plasmatique). Ensuite, leur « voyage» leur permet de croiser des images de la formation des grandes structures de l'Univers, de la création des galaxies, des étoiles, de la collision des galaxies et, plus proche de nous, de se retrouver dans notre galaxie et notre système solaire. Nous nous sommes autorisés à donner une forme artistique à ces différents matériaux du travail scientifique, et, par delà la division du travail et la spécialisation qui a cours dans ces domaines de science, de les agencer dans le cadre d'une aventure vécue par des chercheurs d'aujourd'hui.

Nous revendiquons cette posture qui consiste à travailler avec les énigmes, avec les parties non établies du travail de science. Même s'il s'agit de domaines encore non "stabilisés ", ces grandes questions inspirent la communauté scientifique. Ces énigmes sont des lieux d'engagement, de controverse, d'émulation, de prises de parti, de rebondissements, de passions. Les mettre en image, en histoire, en scène, c'est d'une certaine manière les rendre accessibles au public. Nous pensons aller à la rencontre, ce faisant, d'une forte attente du spectateur. En effet le public est curieux pour cet aspect des sciences en découverte. Il n'attend pas seulement qu'on lui délivre, sur un mode pédagogique, ce qui ne pose plus 
de problème dans le domaine des sciences. Il attend, il souhaite même partager avec le chercheur les questions non résolues, les observations qui font problème, les hypothèses audacieuses, qu'elles soient confirmées ou infirmées. Il attend de la part de la science et de la médiation scientifique un ton qui ne le renvoie pas à son ignorance. Il a envie de vivre avec les chercheurs une relation de découverte, d'hypothèse, d'expérimentation. Il a envie de comprendre que l'Homme dans l'Univers ne comprend pas tout!

\section{Permettre une création indépendante dans les planétariums}

Le monde des planétariums est en profonde mutation, pour des raisons qui tiennent à la fois à l'évolution des outils de projection et de création des spectacles (numérique et diffusion pleine voûte) et également d'une évolution qui tient à la place de ces équipements dans le paysage de la culture scientifique et technique, dans les pratiques du public et dans ses attentes. La visite au planétarium en famille ou avec l'école, comme on va au zoo ou à l'aquarium, ne disparaît pas. Mais elle ne suffit plus à remplir des salles dont linvestissement et le fonctionnement sont assez

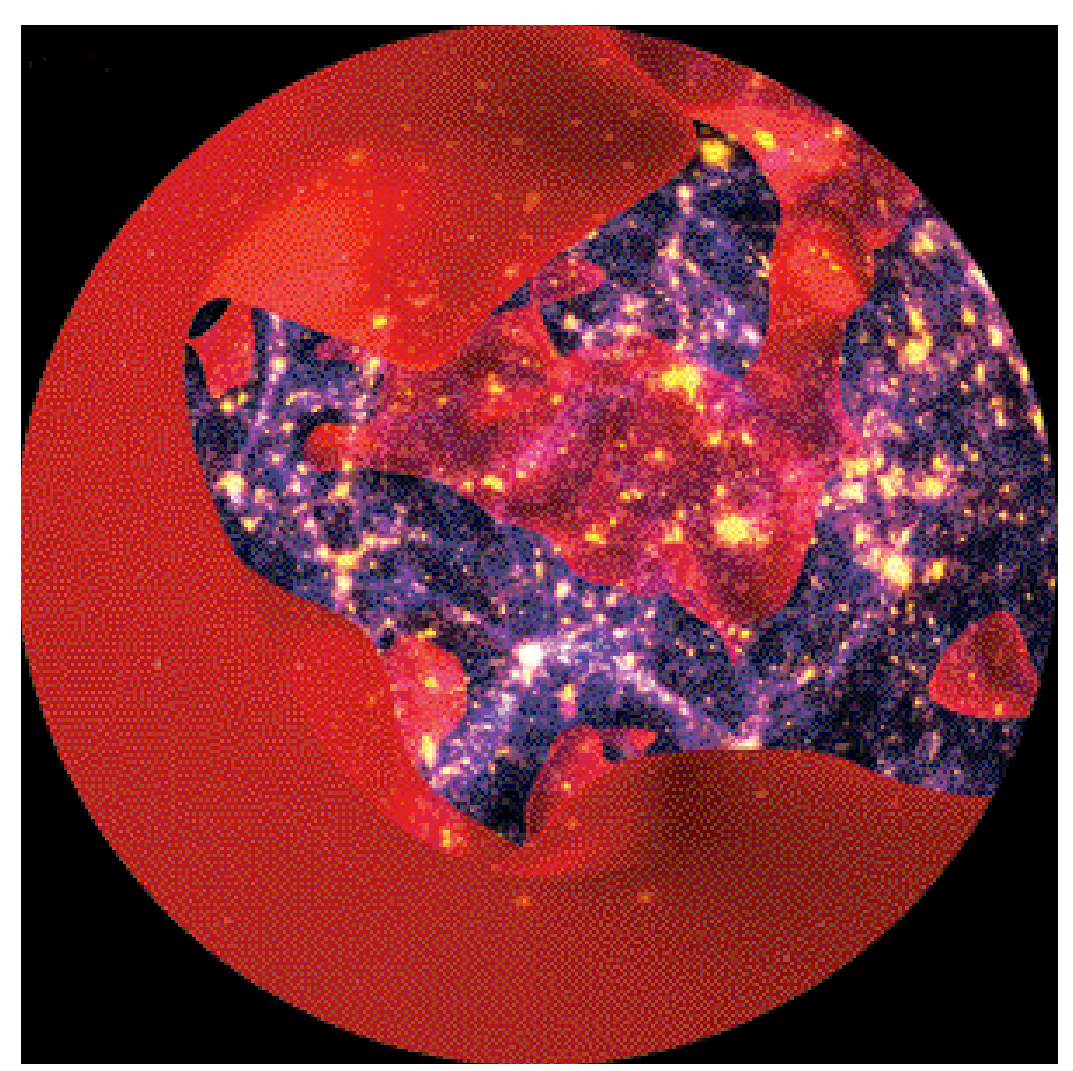

Utilisation de la simulation scientifique : la modélisation des seuils de densité dans les grandes structures de l'Univers ๑) CRAL, CNRS, université Lyon 1/Thierry Sousbie coûteux pour des municipalités qui demandent de plus en plus des comptes et des retours sur investissement. Les formes de visite au planétarium évoluent. Ce qui parait avoir le vent en poupe, ce sont les visites prolongées, dans des lieux conviviaux et interactifs, avec des expositions temporaires, des thèmes, des ateliers. Les grands centres de loisirs scientifiques (Cité des Sciences et de l'Industrie, Cité de l'Espace à Toulouse) disposent de ces ressources de manière naturelle. Différents planétariums tentent de se mettre à l'unisson (Rennes, Vaulx-en-Velin, Montpellier). Le passage dans une séance de spectacle prend place alors dans un parcours, dans un temps consacré à un séjour prolongé. Mais une autre condition de réussite tient à la programmation des spectacles. La diversité des publics, la variation des raisons d'aller au planétarium, imposent une diversification et une inventivité dans l'offre de création des spectacles, et notamment la nécessité de se libérer d'une image trop directement associée au jeune public et à la pédagogie.

Pour parvenir à sa mutation, le milieu des nouveaux planétariums est nécessairement confronté à une transformation profonde des rôles des différents partenaires impliqués dans ce domaine de la culture scientifique. Le marché des systèmes techniques numériques pour planétariums se partage entre trois ou quatre fabricants au niveau mondial (largement dominé par deux firmes américaines et une société française). Or ces fournisseurs d'équipement, notamment parce qu'ils offrent à leurs clients une programmation de lancement et une logistique d'accompagnement quand un nouveau spectacle est créé, sont aussi des distributeurs. Il n'y a pas de distributeur indépendant de spectacles pour planétariums. De plus, et peut-être pour réagir à cette situation de fait, les équipes de planétariums souhaitent également devenir des producteurs de spectacles. Des formules de coopération se font jour avec des échanges entre co-producteurs. Par ailleurs, l'association des planétariums de langue française (APLF) se veut garante d'une certaine forme de morale professionnelle dans l'univers des planétariums. Vibrato, qui résulte d'une coproduction entre un planétarium et une équipe théâtrale, est ici hors des normes, même si sa distribution a été confiée à l'équipementier français RSA. Le collectif qui l'a créé est une hybridation originale entre des auteurs/réalisateurs, une équipe de planétarium inscrite dans une tradition innovante et des scientifiques très ouverts à de nouvelles propositions de relation avec le 
public. Cette configuration inédite dans le milieu des planétariums a quelque chose de " bousculant ». Elle peut provoquer des réactions de surprise, voire de méfiance. Mais elle peut aussi être regardée, à l'inverse, comme une fenêtre ouverte sur des expériences de création indépendantes, qui garantissent aux équipes de planétariums des propositions de spectacles allant au devant des nouvelles aspirations du public. En tout cas, c'est dans cet esprit qu'en tant qu'initiateurs de Vibrato,nous entendons continuer l'aventure à travers d'autres projets.

\section{Conclusion}

La création de Vibrato, la résonance de l'univers, nous a permis de travailler en étroite collaboration avec une équipe de chercheurs et de rencontrer le monde des planétariums « de nouvelle génération ». Elle nous a également permis de nous confronter aux difficultés de l'appropriation de la science, de ses certitudes, de ses énigmes dans le contexte particulier de ces lieux dédiés à la culture astronomique.
Nous nous sommes lancés dans la fiction de Vibrato pour rencontrer le public dans nos fascinations communes, nos interrogations humaines vis-à-vis de la science. Mais si le public rentre dans notre histoire, il apparaît également que notre positionnement quelque peu hors normes pose encore des problèmes pour certains responsables de planétariums ou pour leurs comités scientifiques. Les relations entre la science et la fiction, ou plus globalement son appropriation par des artistes, sont bien ici au cœur du problème. Tout comme les rôles éducatif et distractif d'un équipement de culture scientifique et technique qui dispose désormais d'une formidable machine à faire rêver.

\footnotetext{
Note

(1) « Ce spectacle est une fiction. Les observations astronomiques et les hypothèses de recherche évoquées témoignent simplement des interrogations des astronomes face à la complexité de l'univers ».
} 